\title{
The Anesthetic Management of a Patient with Myasthenia Gravis and Tracheal Obstruction
}

\section{Background}

Tracheal obstruction complicating myasthenia gravis (MG) makes anesthetic management difficult. This case illustrates the steps to follow to deal with this situation and the possibility to make thymectomy at the same surgical time (1).

\section{Case report}

A 40-year-old woman with MG, grade IIB of Osserman classification, required resection of a critical tracheal stenosis. In fact, she was intubated and ventilated during a month for a respiratory distress syndrome complicating her disease and she needed a tracheostomy. Then she developed a tracheal stenosis which was evaluated with tracheal fiberscopy and thoracocervical scanner (fig. 1-2).

During the preparation for surgery, the patient was under anticholinesteratic medication and immunosuppressive therapy (Azathioprine and corticosteroids). Moreover, we planned two sessions of Plasmapheresis. Surgery was done under general anesthesia, using Fentanyl, Propofol and cistracurium with low and effective dose of drugs, and it lasted 3 hours.

First, the ventilation has been achieved through the cuffedtracheostomy tube. After the anesthesia induction, the surgeon replaced the tracheostomy tube by an armored endotracheal tube. Then, an orotracheal tube has been placed below the cords just above the stenosis.

After the tracheal resection, a small jet ventilation catheter (CH12) was inserted through the orotracheal tube. We used High Frequency Jet Ventilation (HFJV) delivering pulses of small jet of gas derived from a high pressure source, 3 bars at a rate of $130, \mathrm{Ti} /$ Ttotal $=30 \%, \mathrm{FiO} 2=100 \%$.

Furthermore, partial thymectomy, through the same cervicotomy, was practiced as a therapeutic action because the MG was partly equilibrated.

Extubation was carried out carefully after a systematic aspiartion with fiberscopy. Cholinesterase inhibitors were resumed again six hours after extubation and Azathioprine the next day.

The postoperative course was marked by the installation of a non-hypoxemic pulmonary disease.

Pseudomonas aeruginosa was identified and the outcome was favorable with targeted antibiotics.

\section{Discussion}

This case illustrates that the combination of $\mathrm{MG}$ and tracheal obstruction presents some difficulties. Treatment involves immune suppression with steroids or azathioprine, boosting neuromuscular transmission with anticholiesteratic drugs, plasma-pheresis, and thymectomy. It is essential to avoid drugs interfering with the neuromuscular junction and controlling the side effects of immuno-suppressive and anticholiesteratic drugs. Intraoperatively, the challenge of the anaesthetist is to accomplish safe management of the airway that is shared with the surgeon. A mini-invasive approach allows to reduce the length of incision and minimize aggressiveness.

The main concerns for anaesthetists are perioperative respiratory and bulbar muscle strength. If there is weakness, the patient is at high risk of aspiration and respiratory distress. Besides, patient with $\mathrm{MG}$ is already vulnerable to infections because of the immunocompromised status.

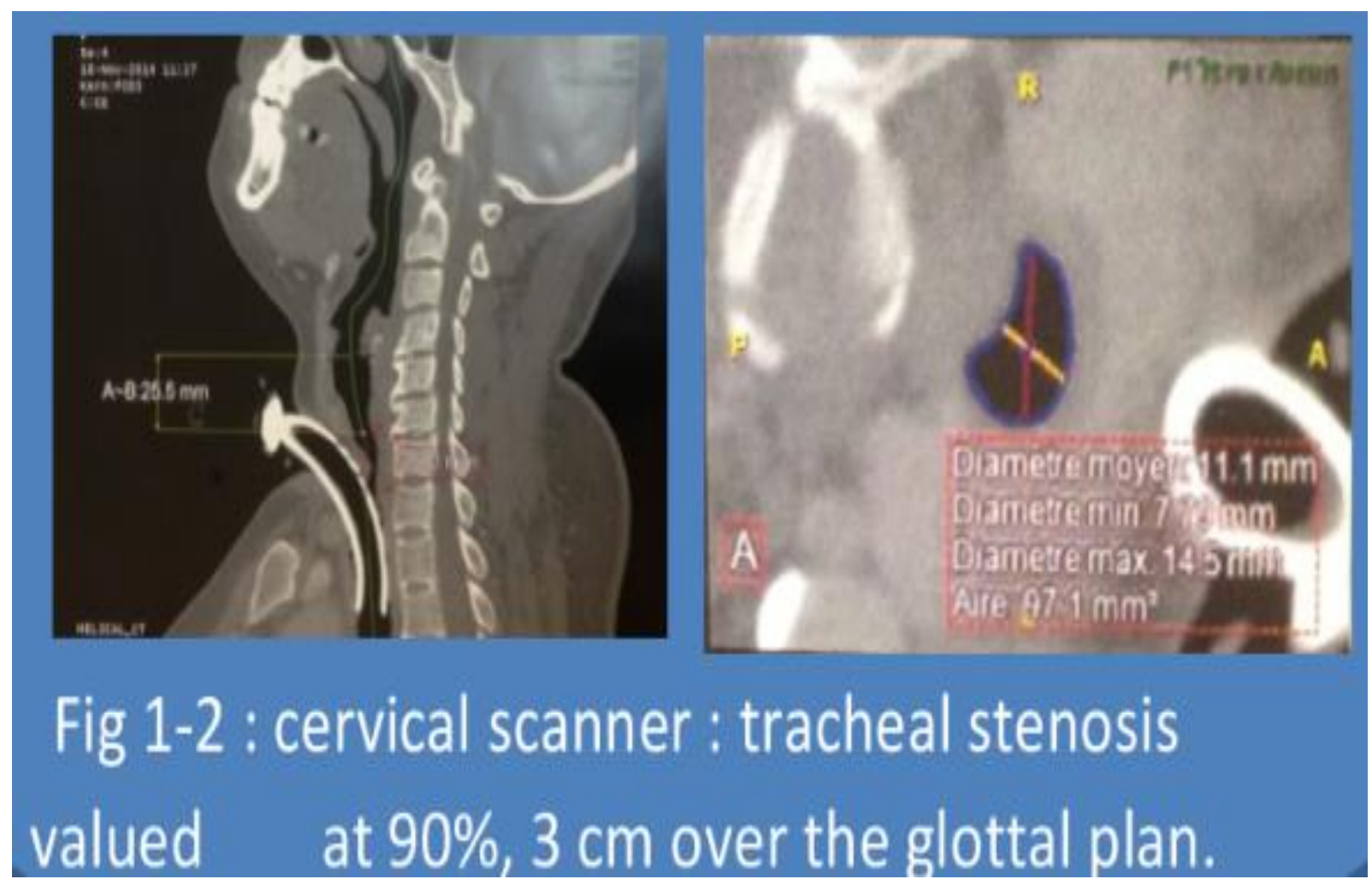

\section{Conclusion}

Planning to operate a patient presenting $\mathrm{MG}$ and tracheal stenosis needs special attention. Thymectomy may produce significant improvement in myasthenic patients and it can be made at the same operative time than the tracheal resection for tracheal stenosis.

\section{References}

1. Jennifer Froelich, Anesthetic management of a patient with myasthenia gravis and tracheal stenosis, Canadian Journal of Anesthesia 1996. 